\title{
Assessing and improving management practices when planning packaging waste collection systems
}

\author{
Tânia Rodrigues Pereira Ramos ${ }^{a}, *$, Maria Isabel Gomes ${ }^{b}$, Ana Paula Barbosa-Póvoac \\ a Instituto Universitário de Lisboa (ISCTE-IUL), Business Research Unit (BRU), Av. das Forças Armadas, 1649-026 Lisboa, Portugal \\ b CMA. Universidade Nova de Lisboa, Campus da Caparica, 2829-516 Caparica, Portugal \\ c CEG-IST, Instituto Superior Técnico, Universidade de Lisboa, Av. Rovisco Pais, 1049-001 Lisboa, Portugal
}

\section{A R T I C L E I N F O}

\section{Article history:}

Received 14 December 2012

Received in revised form 25 October 2013

Accepted 18 December 2013

\section{Keywords:}

Collection routes

Service areas

Routes scheduling

Recycling

Waste management

Vehicle routing

\begin{abstract}
A B S T R A C T
Packaging waste collection systems are responsible to collect, within a geographic area, three types of packaging materials (paper, glass and plastic/metal) that are disposed by the final consumer into special bins. Those systems are often characterized by having a network with multiple depots that act as transfer and sorting stations, and where the vehicle fleet is based. However, each depot is often managed independently and not as a part of a unique system. In this work, four current tactical/operational practices that contribute to the independent management of each depot are analysed. The change of such practices is investigated and their impact assessed on the total collection cost. A solution methodology based on mathematical formulations is developed to plan service areas, vehicle routes and vehicle schedules taking into account new alternative solutions in managing the system as a whole. Such methodology is applied to a real case study of a company responsible for the collection of the packaging waste in 7 municipalities in mainland Portugal. New service areas, collection routes and vehicle schedules are defined and significant savings are obtained in terms of the total distance travelled as well as in terms of the number of required vehicles, resulting in a decreasing of the total system cost.
\end{abstract}

(c) 2013 Elsevier B.V. All rights reserved.

\section{Introduction}

Recycling of packaging materials was imposed by the European Union (EU) to the Members States through the European Community Directive 94/62/EC, which has set targets for recovery and recycling of packaging waste. To meet those targets, a large investment in waste management was made in Portugal since until then all the produced waste was dumped without any kind of treatment. A new collection system - the selective collection - had to be developed given that the traditional routes defined for undifferentiated waste did not fit the particularities of the recycling materials: different vehicles, different collection rates and different bin locations.

The Green Dot System was created in 1996 to promote selective collection, sorting, recovery and recycling of packaging waste in Portugal in order to accomplish the targets set by EU. This system is funded by packers/manufactures who are responsible for products final disposal (according to the Extended Producer Responsibility principle) and had transferred such responsibility to an entity duly licensed for this activity (the Sociedade Ponto Verde (SPV) which manages the Green Dot System). The packers pay a

\footnotetext{
* Corresponding author. Tel.: +351 217903412; fax: +351 217903904.

E-mail addresses: tania.ramos@iscte.pt (T.R.P. Ramos), mirg@fct.unl.pt (M.I. Gomes), apovoa@ist.utl.pt (A.P. Barbosa-Póvoa)
}

"green dot fee" for each package sent to the market according to its weight and materials used. With the revenues from the green dot fees, SPV supports the selective collection and sorting costs. The selective collection and sorting operations are performed by municipal and multi-municipal waste collection companies that are paid according to the weight and type of material delivered to SPV. This financial support value, predetermined by the latter, intends to cover only the costs incurred with collection and sorting operations for the packaging waste materials, deducting the costs avoided with undifferentiated collection and landfill disposal. Therefore, it is crucial that waste collection companies operate their collection and sorting systems in an efficient way, otherwise excessive costs are incurred that will not be refunded. To get more insights on the Green Dot System and on the efficiency of the waste collection companies, please see Marques et al. (2012).

To increase efficiency in recyclable waste collection systems, an optimized solution in terms of service areas, vehicle routes and vehicle schedules should be pursuit. In packaging waste collection systems operating in Portugal, collection costs represent about $66-69 \%$, while sorting costs represent about $11-30 \%$ of the total costs (APA, 2008). Thus, savings in the collection costs are of great impact on the total costs.

The aim of this work is to build new solutions so as to reduce the collection costs by optimizing the service areas, vehicle routes and vehicle schedules under alternative network management 
scenarios. The majority of the recyclable collection systems have more than one depot in the network and it is a current practice to manage each depot independently. Therefore, service areas are defined by depot, vehicles are fixed to a depot and only closed routes are allowed (routes starting and ending at the same depot). This work intends to assess and improve four management practices, commonly used by the packaging waste collection systems operating in Portugal, that involve the following assumptions: (1) respect the municipalities boundaries to define service areas; (2) the service areas are defined by depot; (3) the vehicles are fixed to a depot and can only perform routes starting and ending at that depot; and (4) only closed routes are allowed. To achieve this goal the next questions are raised and consequently analysed: if the systems responsible for the collection and sorting of packaging waste are multi-municipal, why should the geographic boundaries of the municipalities define the service area of each depot? If multiple materials are collected in independent routes, why not having service areas by recyclable material? If the system has multiple depots and all vehicles belong to the company, why not sharing the resources (vehicles) among depots? If the system has multiple depots, why not allow routes to start at a depot and to end at a different one?

The main contribution of the current work is then on assessing the impact of breaking up with the current tactical/operational practices that consider that depots are managed independently and not in an integrated way. For that, we proposed a unified solution methodology that is capable to plan the collection systems exploring more efficient practices. The solution methodology is applied to a real packaging waste collection system and the results are compared with the current solution where the four practices are used.

The remainder of the paper is structured as follows. Section 2 briefly reviews the literature on routing problems. Section 3 describes the main features of the case study. In Section 4 the solution methodology is described and the results of the application to the case study are presented at Section 5. Finally, Section 6 concludes the paper and draws some future research directions.

\section{Literature review}

Recycling has several environmental and economic benefits such as mitigating resource scarcity, decreasing demand for landfill space and involving savings in energy consumption (Craighill and Powell, 1996). However, the activity of recyclable waste collection has also several environmental and economic costs as it is basically a transportation activity. To diminish such costs, collection routes should be defined in order to minimize the total distance travelled or the total routing cost. The problem of defining the optimal collection routes is known in the literature as the Vehicle Routing Problem (VRP). This problem is widely studied and several methods were proposed to solve it in the last decades (see the recent surveys of Golden et al., 2008; Laporte, 2009). However, the routing problem that appears in the packaging waste collection systems goes far beyond the classical VRP. The vehicle fleet is based at multiple depots, multiple products have to be collected and different collection frequencies are observed for each product and site. Therefore, the routing problem arising in the packaging waste collection systems is the Multi-Product, Multi-Depot Periodic Vehicle Routing Problem (MP-MDPVRP), where it has to be decided from which depot the multiple products at each site should be collected, in which day of the planning horizon each site should be visited and what should be the collection visit sequence in order to minimize the total routing cost. Parthanadee and Logendran (2006) presented a mathematical model for the MP-MDPVRP and three tabu search heuristics are developed to solve it. However, the authors had called the problem "multi-product" as several products have to be delivered to customers, but the same customer can be visited by vehicles from different depots. In our case, "multi-product" refers to the fact that the three packaging materials in each site have to be collected from the same depot.

Considering only one product, the MDPVRP has been studied by Hadjiconstantinou and Baldacci (1998) and recently by Vidal et al. (2012). In the former work, a heuristic approach based on tabu search has been developed. The heuristic algorithm is applied to a real case of a utility company that provides preventive maintenance services to a set of customers. This company has 17 vehicles, based on 9 depots, to serve 162 customers with a frequency that can vary from once a day to once every four weeks. The large scale problem motivated the authors to apply a heuristic algorithm instead of an exact one. In the latter work, the authors proposed a hybrid genetic algorithm with adaptive diversity control to solve several classes of multi-depot and periodic vehicle routing problems, including the MDPVRP, and applied it to several test instances.

Considering only one depot, several heuristic approaches have been developed for the PVRP where a planning horizon of several days is considered as customers have different visiting frequencies. Beltrami and Bodin (1974), Russel and Igo (1979) and Teixeira et al. (2004) developed heuristic algorithms for the PVRP which were applied to waste collection problems. Mourgaya and Vanderbeck (2007) presented a column generation procedure followed by a rounding heuristic to solve a PVRP with two objectives: minimizing total distance travelled and balance workload among vehicles. Other heuristic applications to PVRP can be found in Christofides and Beasley (1984), Gaudioso and Paletta (1992), Chao et al. (1995), Cordeau et al. (1997) and Alonso et al. (2008), to name a few.

Considering a single time unit planning horizon with multiple depots, several works have been published to tackle the MDVRP. Golden et al. (1977), Salhi and Sari (1997), Cordeau et al. (1997), Thangiah and Salhi (2001), Lim and Wang (2005), Lau et al. (2010) are some works where heuristics and meta-heuristics approaches were developed. Laporte et al. $(1984,1988)$ and Baldacci and Mingozzi (2009) have proposed exact methods to solve the MDVRP. Among the works on multi-depot problems, we highlight one where inter-depot routes are considered. Crevier et al. (2007) study an extension of the MDVRP in which vehicles may be replenished at intermediate depots along their route (Multi-Depot Vehicle Routing Problem with Inter-Depots Routes). The authors propose a heuristic combining the adaptative memory principle and a tabu search method for the generation of a set of routes, and an integer programming model in the execution of a set partitioning algorithm for the determination of the least cost feasible rotations (the authors define rotation as the set of routes assigned to a vehicle).

In waste collection problems, there is another critical aspect to address: the estimation of the waste amount to collect at each bin. The majority of works uses deterministic input data. However, recent works like Johansson (2006), Faccio et al. (2011) and Anghinolfi et al. (2013) have approached dynamic routing in waste collection problems, where modern traceability devices, like volumetric sensors, identification RFID (Radio Frequency Identification) systems, GPRS (General Packet Radio Service) and GPS (Global Positioning System) technology, permit to obtain data in real time, which is fundamental to implement an efficient routing plan. The basic idea is that, if the real time position and replenishment level of each vehicle are known, as well as the real time waste level at each bin and which bins have been visited, it is possible to decide which bins should be emptied and which can be avoided at a certain time. This allows an optimization of the route plan and to minimize covered distance and number of vehicles needed, which, as a consequence, would minimize travel time, number of load-unload stops, exhaust emissions, noise and traffic congestion (Faccio et al., 2011). 


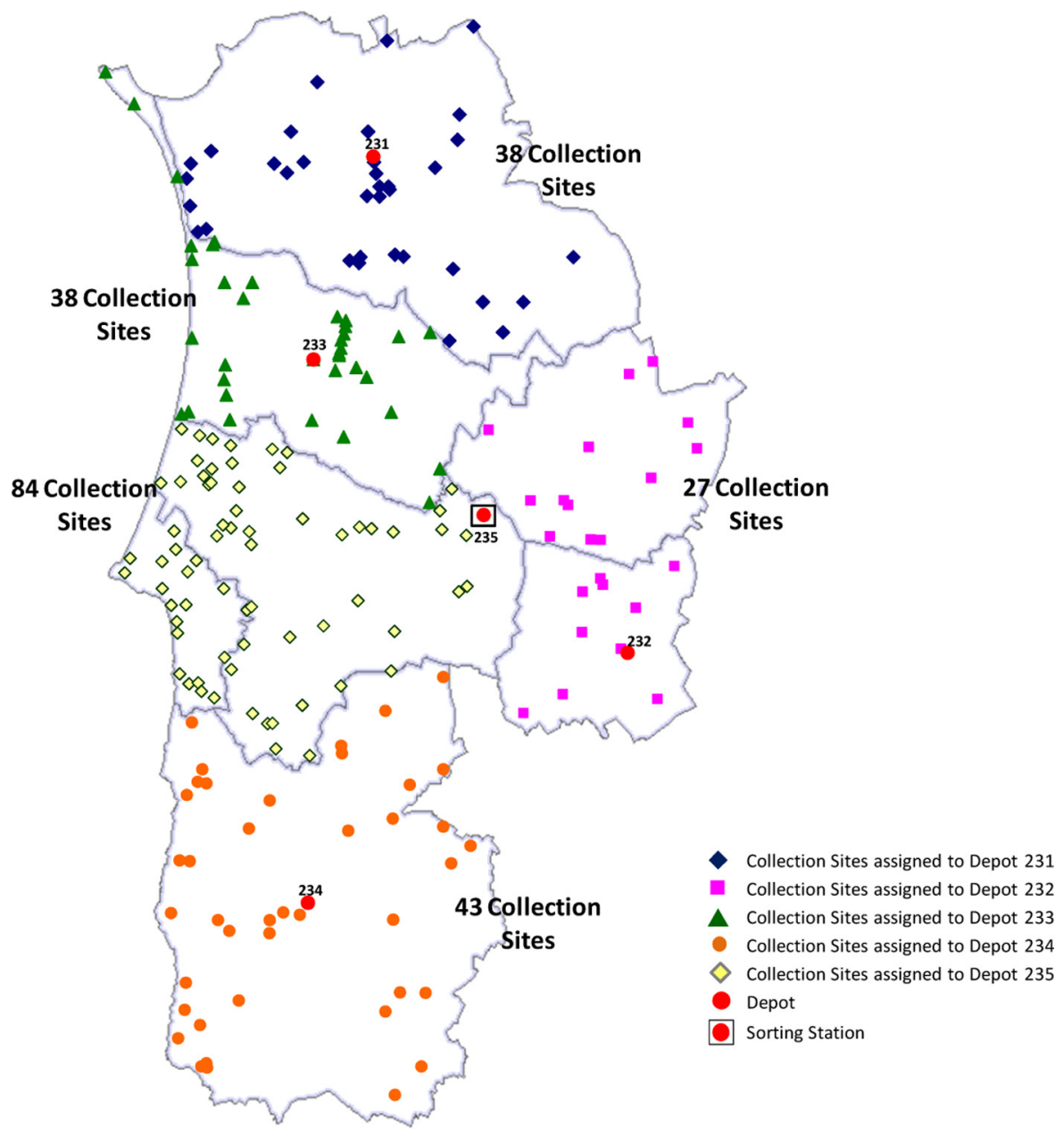

Fig. 1. Depot's location and current service areas.

\section{Case study - a real packaging waste collection system}

In Portugal there are 31 recyclable collection systems (SPV, 2011), each one responsible for a certain number of municipalities (Portugal has a total of 308 municipalities). Our case study focuses on the company responsible for the recyclable collection system covering seven rural municipalities with a total area of $6400 \mathrm{~km}^{2}$. This company operates five depots where the collection vehicles are based. One of the depots operates also as a sorting station (depot 235, see Fig. 1). The remaining four are only transfer stations where the packaging waste is consolidated and afterwards transferred to the sorting station. Given such logistics network configuration, two types of transportation flows need to be considered, namely, the inbound flow from the collection sites to the depots and the outbound flow from the depots to the sorting station. The collection is performed by a vehicle fleet with no compartments, so each packaging material has to be collected in separated routes. There are 651 Glass bins, 513 Paper bins and 458 Plastic/Metal bins spread over 230 localities (see Fig. 1). It is assumed that a collection site corresponds to a locality instead of an individual container in order to reduce the problem size. Due to the proximity of the containers within a locality (an average distance of $650 \mathrm{~m}$ is observed) it is practicable to treat the containers to collect within a locality as a single node. Therefore, a collection site aggregates one or more containers of one or more recyclable materials, meaning that the distance travelled and the time spent within a collection site have to be considered.
All the collection routes start at a depot, visit several localities collecting a single type of material, and return to a depot to unload. Collection is performed five-days a week, $8 \mathrm{~h}$ per day, during daytime. It is assumed that the collection sites can be collected at any time of the day within the working period, i.e., between 10 a.m. and 19 p.m. (with a lunch break of an hour). No time windows are considered for each site.

The three recyclable materials have different collection frequencies. Glass has to be collected once a month, Plastic/Metal every two weeks and Paper every week. Therefore, each route has to be scheduled in a four-week planning horizon that is to be repeated every four weeks. Due to vehicle volume capacity constraints and taking into account each material's density, vehicles can load a maximum of $4500 \mathrm{~kg}$ of Glass, $3400 \mathrm{~kg}$ of Paper and $600 \mathrm{~kg}$ of Plastic/Metal. For the outbound transportation, i.e., from the depots to the sorting station, larger vehicles are used where weight capacities are increased to $12,000 \mathrm{~kg}$ for Glass, $4000 \mathrm{~kg}$ for Paper and $2000 \mathrm{~kg}$ for Plastic/Metal. Furthermore, one of the company policies is that each depot is responsible for a fixed set of containers, thus there is a need of defining the depots service areas. Nowadays, the company operates service areas considering the municipalities' boundaries. Moreover, all recyclable materials at each collection site have to be collected from the same depot, meaning that each depot has only one service area common to all recyclable materials. The current service areas are depicted at Fig. 1, which together with the current vehicle routing plan imply an average of $28,000 \mathrm{~km}$ driven per month (four weeks) and a vehicle fleet of 9 vehicles. 


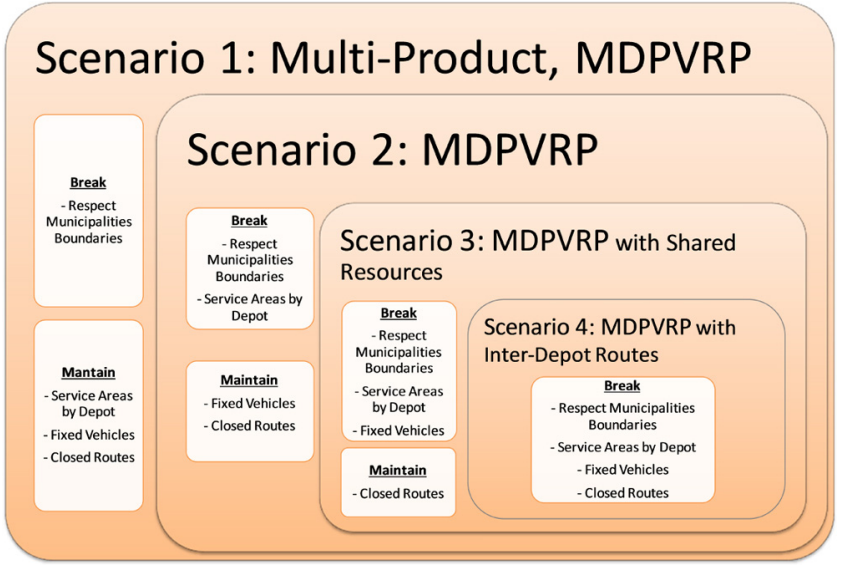

Fig. 2. Scenario's description.

Given the current solution's cost, the company envisages the restructure of their service areas and vehicle routes plan in order to decrease such value. An optimized plan is pursued where service areas, vehicle routes and route scheduling can be redefined in a breakthrough scenario, i.e., where service areas do not have to respect municipalities boundaries, can be defined by packaging material, resources can be shared among the depots and open routes between depots are allowed.

\section{Solution methodology}

Accordingly to the four practices used by the packaging waste collection system operating in mainland Portugal, four scenarios will be study wherein each practice is broken cumulatively until the breakthrough scenario is reached (see Fig. 2). The first scenario breaks with the practice "respect municipalities boundaries" and maintains the remaining three. In this case, the problem to be solved is the Multi-Product, Multi-Depot Periodic Vehicle Routing Problem (MP-MDPVRP). The second scenario breaks with two practices, "respect municipalities boundaries" and "service areas by depot", and maintains the remaining two. In this case each packaging material is solved independently. Therefore, a Multi-Depot Periodic Vehicle Routing Problem (MDPVRP) for each packaging material has to be solved. The third scenario breaks with an additional practice, "vehicles are fixed to a depot" while maintaining the "closed routes" practice. Here a MDPVRP with Shared Resources is solved, where the vehicle based at one depot can perform collection routes starting and ending at a different depot. In this scenario, only one relocation movement is allowed per vehicle, i.e., a vehicle based at depot $i$ can travel to depot $j$ to perform closed routes from depot $j$ and then returns to home depot $i$. It is not allowed two or more relocation movements, such as: a vehicle based at depot $i$ travelling to depot $j$ and then to depot $h$ and returning to depot $i$. Finally, in the fourth scenario, the breakthrough scenario, the four practices are broken and a MDPRVP with Inter-Depot Routes is solved. At this

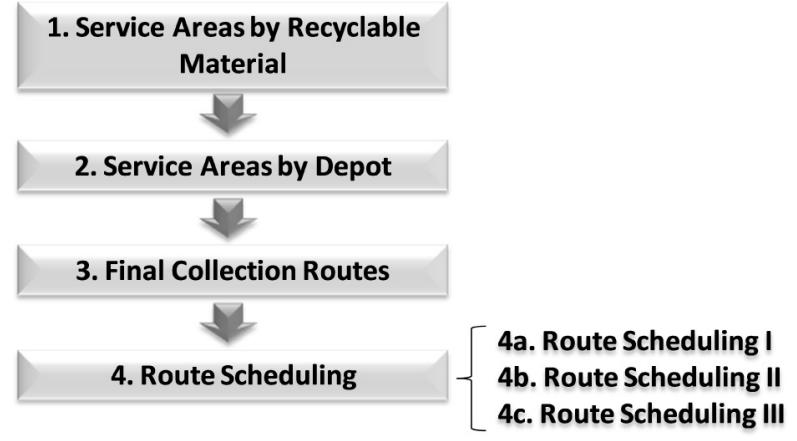

Fig. 3. Solution methodology main modules.

point open routes between depots are allowed but, at the end of a working day, the vehicles have to return to their home depot.

To solve the above scenarios we proposed a solution methodology to define service areas, vehicle routes and schedules. This approach involves four main modules, as it is shown at Fig. 3. The first module defines service areas by recyclable material, the second module defines service areas by depot, the third module defines the final collection routes and the fourth module defines vehicle schedules according to the characteristics of each scenario. This solution methodology is an extension of the method proposed in Ramos et al. (2014) where only service areas and vehicle routes are defined for the MP-MDVRP. The periodic issue (routes scheduling), shared resources and open routes are not tackled in the mentioned work.

For scenario 1, where a MP-MDPVRP is solved, modules 1-3 define the service areas and the final vehicle routes for each depot and module 4a schedules, within the planning horizon, the routes generated by module 3 (see Fig. 4). For scenario 2, where a MDPVRP is solved, module 1 defines the service areas for each packaging material, module 3 defines the final collection routes and module 4a schedules the routes. For scenarios 3 and 4, only the scheduling module differs from scenario 2 . At scenario 3 , the scheduling module (module $4 \mathrm{~b}$ ) takes into account that the vehicles can be shared among depots. At scenario 4, besides taking into account a sharedresources solution, the scheduling module (module 4c) considers also all the vehicle routes defined along the solution procedure (open and closed routes defined by module 1 ) together with the vehicle routes defined at module 3 .

Each module of the solution methodology involves mathematical formulations. Each module will be detailed in the following section.

\subsection{Modules description}

\subsubsection{Module 1: service areas by recyclable material}

To define service areas by recyclable material, a MDVRP has to be solved for each material. As mathematical programming solvers cannot solve large size instances of the MDVRP, we adopted the solution method developed in the work of Ramos et al. (2014) as

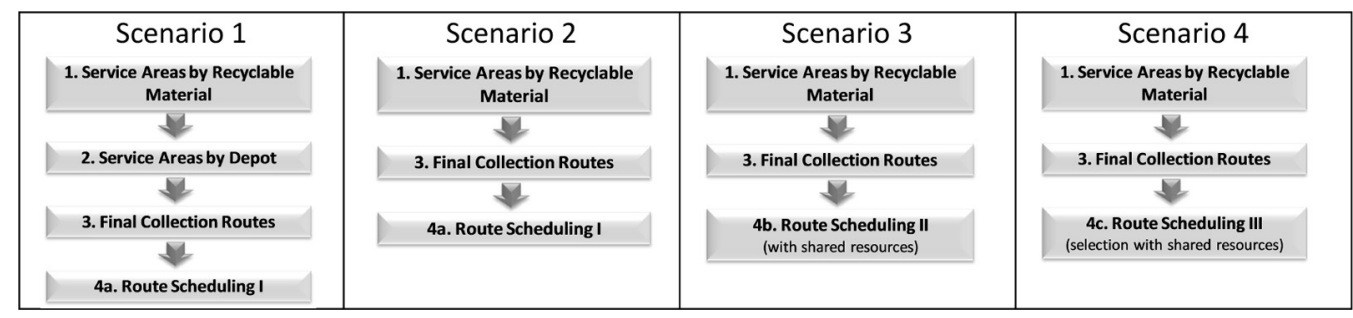

Fig. 4. Solution methodology for each scenario. 


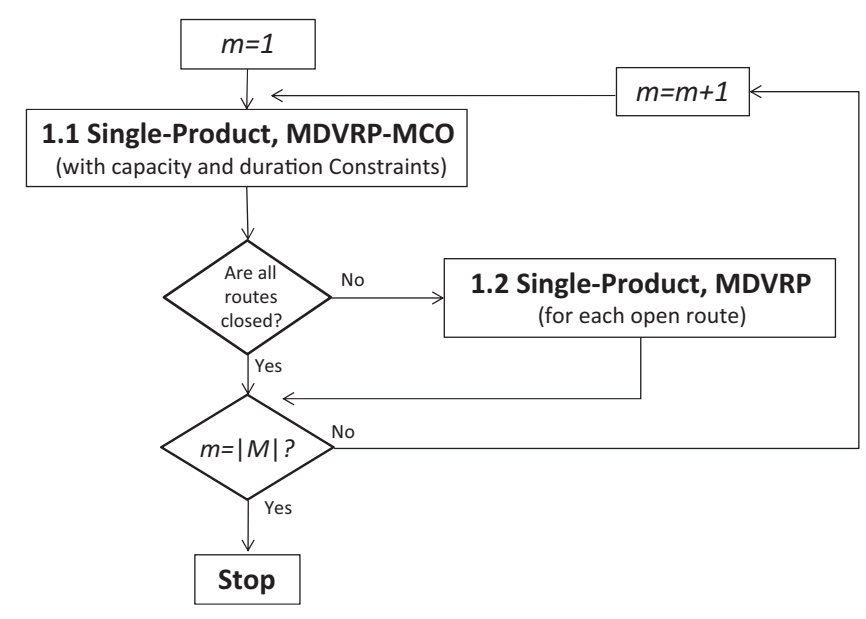

Fig. 5. Solution method to solve the MDVRP.

shown in Fig. 5. Notice that $m$ represents the packaging materials, and $|M|$ the total number of materials to be collected.

Firstly, it is solved a Single-Product, MDVRP with Mixed Closed and Open Routes (MDVRP-MCO). This problem involves the definition of the optimal routes to collect one single product, considering a network with multiple depots and allows closed and open routes between depots. As input data, this module requires the distance between each node (collection sites and depots), the weight to be collected and the service time at each collection site (considering only one recyclable material), the vehicle capacity and the maximum time allowed for a working day. The output will be a set of collection routes, where some routes start and end at the same depot (closed routes) while others start and end at different depots (open routes). Since this formulation allows closed and open routes between depots, it should be guarantee that the number of vehicle routes starting and ending at each depot is the same. Note that the number of vehicle routes is not constrained because the vehicle fleet size is one of the model's outputs. For further details see Ramos et al. (2013).

If open routes are produced, a MDVRP is solved to close them so that service areas are defined. An open route links two different depots, meaning that collection sites within such route are assigned to two depots instead of just one. By solving a MDVRP to the collection sites that integrate each open route, closed routes are obtained.

Considering the packaging material set $M$, this solution method have to be run for all materials included in set $M$.

\subsubsection{Module 2: service areas by depot}

If service areas by depot are required, module 2 is executed (this will only be of use in scenario 1 ). Service areas by depot imply that all packaging materials at each collection site are collected from the same depot. Comparing the service areas defined for each packaging material in the previous module, some sites may not respect that rule. Such sites are named as "unclear sites" since there is no agreement among the packaging materials concerning their depot assignment. For those sites, a Multi-Product, MDVRP is solved, where a constraint ensures that all materials at each collection site are allocated to the same depot. This formulation is detailed in Ramos et al. (2014).

The mathematical formulation for the Multi-Product, MDVRP is only capable of solving small instances, i.e., problems with a small number of unclear sites (lower than 30 unclear sites, according to preliminary tests). Therefore, one way to reduce the problem size is to consider the number of unclear sites between each pair of depot rather than considering all unclear sites at once. For example, a site

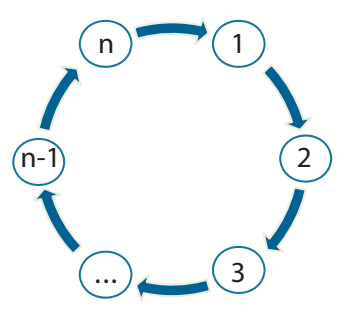

Fig. 6. Planning horizon representation using a directed cycle graph with $n$ vertices.

is "unclear" if it is collected from depot 1 for material Glass and from depot 2 for materials Paper and Plastic/Metal. Therefore, this site is unclear between depots 1 and 2. Since this lack of "clearance" concerns at least two depots, such partition of the unclear sites set would be a natural way to do so. If, with such decomposition, the number of unclear sites for each pair of depots is still above 30 , a heuristic assignment rule has to be defined. In this case, the unclear sites are placed according to the assignment made by the material with the highest collection frequency. Some effectiveness tests performed in the work of Ramos et al. (2014) support such heuristic rule.

\subsubsection{Module 3: final collection routes}

After defining the service areas (by depot or by recyclable material), module 3 is executed to set the final vehicle routes for each depot and each packaging material. Along with modules 1 and 2 vehicle routes are defined, but the aim of those modules is to establish service areas through the definition of the vehicle routes. Therefore, the defined routes can be improved once the final service areas are set, and this is what module 3 is designed for. The CVRP formulation proposed by Baldacci et al. (2004) is extended to deal with duration constraints.

\subsubsection{Module 4: route scheduling}

Finally, the scheduling module defines for each day of the planning horizon which route should be performed, ensuring that the collection frequency over the planning horizon is fulfilled and a minimum and a maximum time interval between consecutive collections is observed in order to prevent containers overflow. Moreover, it is desirable that the same route is performed by the same vehicle/driver.

Each route $k \in K$ (defined in the previous module) is characterized by (1) distance $d_{k}$; (2) duration $b_{k}$, which includes travel, service and unloading times; and (3) load $L_{k}$. Let $K_{m}$ be the routes subset for material $m$. The collection sites belonging to route $k$ are given by a binary parameter $\mu_{i k}$ that equals 1 if collection site $i \in V_{c}$ belongs to route $k$; and 0 otherwise $\left(V_{c}\right.$ is the node set of collection sites). The starting and ending depots for route $k$ are also modelled by binary parameters $S_{k i}$ and $E_{k i}$, respectively: $S_{k i}$ equals 1 if route $k$ starts at depot $i \in V_{d}$ while $E_{k i}$ equals 1 if route $k$ ends at depot $i \in V_{d}$ ( $V_{d}$ is the node set for depots).

Let $G$ be the vehicle set. As vehicles are fixed at the depot, consider the binary parameter $\alpha_{g i}$ equals to 1 if vehicle $g$ belongs to depot $i$; and 0 otherwise.

The collection frequency of each collection site $i$ with recyclable material $m$ is given by $f_{\text {im }}$ representing how often a collection site has to be visited during the planning horizon. The minimum and maximum interval between two consecutive collections for packaging material $m$ are given by $I_{m}$ and $A_{m}$, respectively. Given the periodic nature of the problem, the schedule solution is to be repeated over the next period. Therefore, the planning horizon defined by a discrete set such as $T=\{1, \ldots, n\}$ is here considered to be cyclical and modelled as a directed cycle graph of size $n, C_{n}$, based upon graph theory (see Fig. 6). 
The scheduling model has a single set of decision variables $x_{k t g}$. These are binary variables that equal 1 if route $k$ is performed on day $t$ by vehicle $g$ and equals 0 otherwise.

The scheduling module involves three variants: module $4 \mathrm{a}$, where the routes defined at module 3 are scheduled and the vehicle are fixed at each depot; module $4 \mathrm{~b}$, where the routes defined at module 3 are scheduled, but the vehicles can be shared among depots; and module 4c, where the vehicles can be shared but the set of routes to be scheduled is augmented with the routes defined along module 1 . For module $4 \mathrm{a}$, the objective function is given by Eq. (1), while Eq. (2) models the objective function of modules $4 \mathrm{~b}$ and $4 \mathrm{c}$.

$$
\begin{aligned}
& \text { Min } \sum_{k \in K} \sum_{t \in T} \sum_{g \in G} d_{k} x_{k t g}+\sum_{j \in V_{s}} \sum_{i \in V_{d}} \sum_{m \in M} \sum_{k \in K_{m}} \sum_{t \in T} \sum_{g \in G} \frac{E_{k i} x_{k t g} L_{k}}{O_{m} 2 d_{i j}} \\
& \text { Min } \sum_{k \in K} \sum_{t \in T} \sum_{g \in G} d_{k} x_{k t g}+\sum_{j \in V_{s}} \sum_{i \in V_{d}} \sum_{m \in M} \sum_{k \in K_{m}} \sum_{t \in T} \sum_{g \in G} \frac{E_{k i} x_{k t g} L_{k}}{O_{m} 2 d_{i j}} \\
& \quad-\sum_{j \in V_{s}} \sum_{i \in V_{d}} \sum_{m \in M} \sum_{k \in K_{m}} \sum_{t \in T} \sum_{g \in G} \frac{\alpha_{g j} S_{k i} E_{k i} x_{k t g} L_{k}}{O_{m} 2 d_{i j}} \\
& +\sum_{g \in G} \sum_{k \in K} \sum_{t \in T} \sum_{i, j \in V_{d}} 2 \alpha_{g i} S_{k j}\left(1-E_{k i}\right) x_{k t g} d_{i j}
\end{aligned}
$$

Eq. (1) considers the distance to be travelled (inbound and outbound distance) when vehicles are fixed at depots and cannot be shared. The outbound distance (second term) considers the number of round-trips between the sorting station and the depots modelling transfer of all materials collected by each depot $\left(V_{s}\right.$ is the sorting stations set). To compute the required number of roundtrips, one considers the total load collected by a depot and the vehicle capacity for outbound transportation for each material $m$ $\left(O_{m}\right)$. Note that the number of round-trips is not round upward due to the finitude of the modelled planning horizon. In fact, the actual management of such a system, round-trips are repeated at all time periods.

Eq. (2) considers that although each vehicle belongs to a depot, vehicles can be shared, meaning that they can perform routes assigned to a different depot. Therefore, the objective function needs to account for: (i) the distance to be travelled between depots when a vehicle belonging to depot $i$ is performing routes of depot $j$ (fourth term of Eq. (2)); (ii) the distance related to the outbound transportation when a vehicle based at the sorting station performs routes assigned to a different depot (third term). In the latter case, the load collected will now be unloaded at the sorting station and not at the transfer depot, so the corresponding outbound distance should not be accounted in the objective.

Some constraints are then to be considered in the scheduling models:

$$
\sum_{k \in K_{m}} \sum_{t \in T} \sum_{g \in G} x_{k t g} \mu_{i k}=f_{i m} \quad \forall i \in V_{c}, \quad \forall m
$$

Constraint (3) ensures that a collection site $i$ with material $m$ has to be collected $f_{i m}$ times over the planning horizon.

$$
\begin{gathered}
\sum_{k \in K} x_{k t g} b_{k}+\sum_{k \in K} \sum_{\substack{j \in V_{d} \\
j \neq i}} 2 \alpha_{g i} S_{k j}\left(1-E_{k i}\right) x_{k t g} v_{i j} \leq H \quad \forall t, \quad \forall g, \quad \forall i \in V_{d} \\
j \neq i
\end{gathered}
$$

Constraint (4) states that the route total duration performed by vehicle $g$ on day $t$ will not exceed the maximum number of working hours per day $(H)$. If a vehicle $g$, belonging to depot $i$, performs a route starting at depot $j$, the travel time between $i$ and $j$ $\left(v_{i j}\right)$ is accounted. It is assumed that sites can be collected any time during the working day, i.e., no time windows are being considered concerning the collection sites.

$\sum_{g \in G_{t^{\prime}} \in P_{I_{m}+1}^{t}} x_{k t^{\prime} g} \mu_{i k} \leq 1 \quad \forall i \in V_{c}, \quad \forall k \in K_{m}, \quad \forall m, \quad \forall t$

Constraint (5) states that the same route for material $m$ has to be performed with a minimum time interval of $I_{m}$. This constraint ensures that no more than one visit will occur in any $I_{m}+1$ consecutive days. This is modelled defining $I_{m}+1$ consecutive days as a path of length $I_{m}+1$ of the cycle graph $C_{n}$. In detail, $P_{I_{m}+1}^{t} \subseteq C_{n}$ defines the set of $I_{m}+1$ consecutive days starting at day $t$, in a rolling horizon.

$\sum_{g \in G_{t^{\prime} \in P_{A m}^{t}}} x_{k t^{\prime} g} \mu_{i k} \geq 1 \quad \forall i \in V_{c}, \quad \forall k \in K_{m}, \quad \forall m, \quad \forall t$

Constraint (6) assures the maximum interval $A_{m}$ between consecutive collections. Thus, at least one visit must occur within $A_{m}$ consecutive days.

$P_{A_{m}}^{t} \subseteq C_{n}$ defines the set of $A_{m}$ consecutive days starting at day $t$, in a rolling horizon.

Fig. 7 shows an illustrative example on how constraints (5) and (6) work. Consider a cycle of 20 days, $I_{m}=3$ days and $A_{m}=8$ days. Constraint (5) ensures that at most one collection may occur within four consecutive days. If site $i$ is collected at day 1 , it cannot be collected at days 2,3 and $4\left(P_{4}^{1}\right)$, neither at days 18,19 and $20\left(P_{4}^{18}\right)$. Constraint (6) ensures that within 8 consecutive days, at least one collection must occur. If site $i$ is collected at day 1 , a second collection must occur between day 2 and day $9\left(P_{8}^{2}\right)$. Assuming site $i$ has to be collected four times during the planning horizon $\left(f_{i m}=4\right)$, Fig. 7 shows then one possible schedule.

$x_{k t g}+x_{k t^{\prime} g^{\prime}} \leq 1 \quad \forall k, \quad \forall g, \quad g^{\prime} \in G, \quad g \neq g^{\prime}, \quad \forall t, \quad t^{\prime} \in T, \quad t \neq t^{\prime}$

Constraint (7) ensures that the same route has to be performed by the same vehicle along the planning horizon. Therefore, the collection sites are always visited by the same vehicle and driver.

$x_{k t g} \in\{0,1\} \quad \forall k \in K, \quad \forall t \in T, \quad \forall g \in G$

Variables domain is given in constraint (8).

Constraints (3)-(8) are applied in modules $4 \mathrm{a}$ and $4 \mathrm{~b}$. For module 4c, constraints (9)-(11) should be added because there are alternative routes to be selected, given that all routes defined along the solution procedure are considered in the scheduling module.

$\sum_{k \in K_{o}} S_{k i} E_{k j} x_{k t g}=\sum_{k^{\prime} \in K_{o}} S_{k^{\prime} j} E_{k^{\prime} i} x_{k^{\prime} t g} \quad \forall g, \quad \forall t, \quad \forall i, \quad j \in V_{d}, \quad i \neq j$

Constraint (9) guarantees that all vehicles return to their home depot in case open routes are selected as part of the solution $\left(K_{o}\right.$ is the subset of open routes). If an open route $k$ starting at depot $i$ and ending at depot $j$ belongs to the solution, one open route $k$ ' starting at depot $j$ and ending at depot $i$ must also integrate the solution.

$$
\begin{aligned}
& \sum_{g \in G_{t^{\prime} \in P_{I_{m+1}}^{t}}} x_{k t^{\prime} g} \mu_{i k}+\sum_{g \in G_{t^{\prime}} \in P_{I_{m}+1}^{t}} x_{k^{\prime} t^{\prime} g} \mu_{i k^{\prime}} \leq 1 \quad \forall i \in V_{c}, \\
& \forall k, \quad k^{\prime} \in K_{m}, \quad k \neq k^{\prime}, \quad \forall m, \quad \forall t
\end{aligned}
$$

In case of module $4 \mathrm{c}$, the consecutive collections can be performed by the same route or by two different routes. Constraint (10) ensures the minimum time interval in case two different routes are 


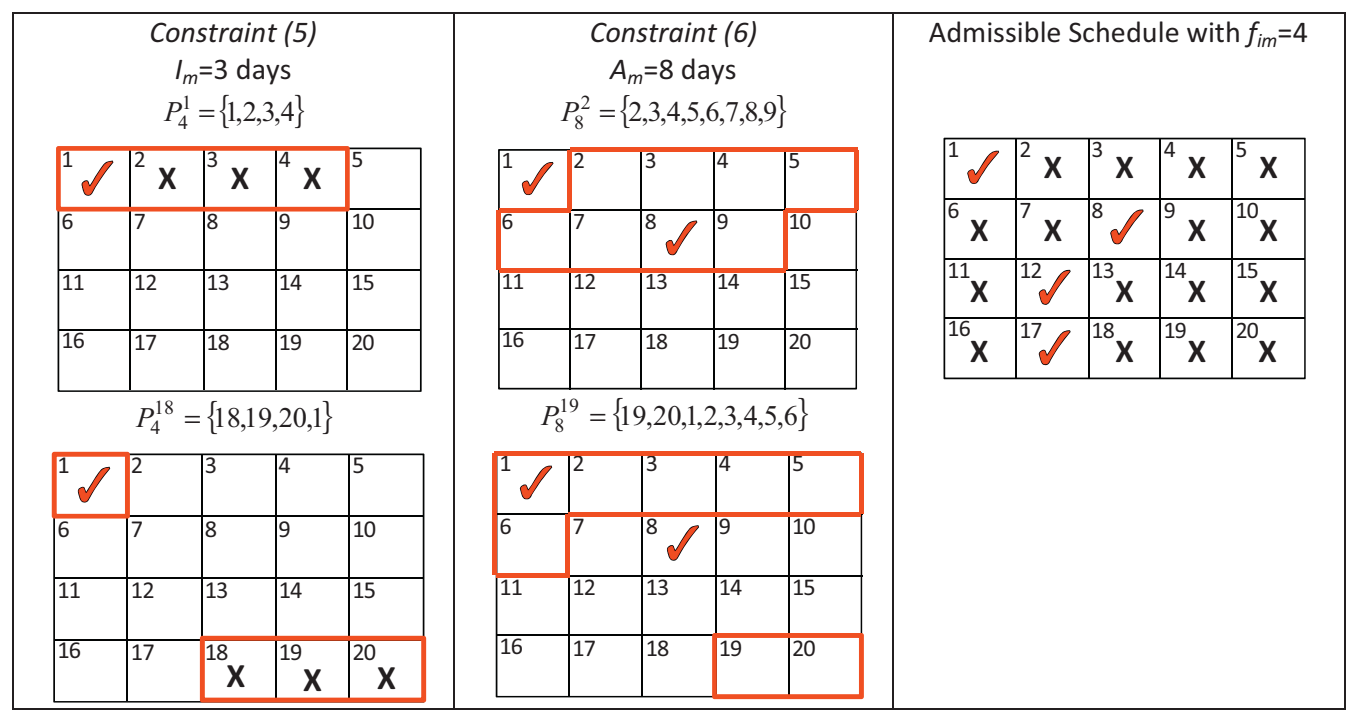

Fig. 7. Illustrative example on how constraints (5) and (6) operate.

collecting the same site $i$, at consecutive collections.

$$
\begin{gathered}
\sum_{g \in G} \sum_{t^{\prime} \in P_{A_{m}}^{t}} x_{k t^{\prime} g} \mu_{i k}+\sum_{g \in G_{t^{\prime} \in P_{A m}^{t}}} x_{k^{\prime} t^{\prime} g} \mu_{i k^{\prime}} \geq 1 \quad \forall i \in V_{c}, \\
\forall k, \quad k^{\prime} \in K_{m}, \quad k \neq k^{\prime}, \quad \forall m, \quad \forall t
\end{gathered}
$$

Constraint (11) guarantees the maximum interval $A_{m}$ between consecutive collections when two different routes collect the same site $i$.

The output of the scheduling modules is a schedule for each vehicle establishing, in each day, the routes to be performed.

\section{Results analysis}

The solution methodology proposed is applied to the packaging waste collection system described at Section 3 in order to plan new service areas, new collection routes and new vehicle schedules, while assessing, regarding the total collection cost, the impact of breaking up with the current practices.

The mathematical formulations developed are implemented in GAMS 23.7 and solved through the CPLEX Optimizer 12.3.0, on an Intel Xeon CPU X5680 @ 3.33 GHz.

The results for each scenario will be shown, followed up by a cost-analysis considering the distance travelled and the number of vehicles required, assessing the impact of each current practice.

\subsection{Scenarios results}

\subsubsection{Scenario 1: municipalities' boundaries}

The first module is run for each one of the three packaging materials: Glass, Paper and Plastic/Metal. The results for the MDVRP-MCO (formulation 1.1) are shown at Table 1.

The difference in the total distance travelled among the three materials is explained by the collection frequency of each material in the planning horizon. Glass has to be collected once, while Plastic/Metal twice and Paper has to be collected four times. On the other hand, Plastic/Metal is the material with the lowest density among the materials, and thus the vehicle weight capacity for such material is smaller for the same vehicle volume capacity. Therefore, more routes are required, and consequently, more distance is travelled.
Table 1

Results for formulation 1.1.

\begin{tabular}{lcllc}
\hline Packaging material & $\begin{array}{l}\text { Total } \\
\text { distance }(\mathrm{km})\end{array}$ & $\begin{array}{l}\text { No. closed } \\
\text { routes }\end{array}$ & $\begin{array}{l}\text { No. open } \\
\text { routes }\end{array}$ & $\begin{array}{l}\text { Total no. } \\
\text { routes }\end{array}$ \\
\hline Glass & 3381 & 18 & 13 & 31 \\
Paper & 11,595 & 21 & 0 & 21 \\
Plastic/metal & 8047 & 38 & 11 & 49 \\
Total & 23,023 & 77 & 24 & 101 \\
\hline
\end{tabular}

Regarding Paper, only closed routes are proposed in the final solution, thus there is no need to solve the MDVRP (formulation 1.2). This can be explained by the fact that there is a little difference between the inbound and outbound vehicle's capacity for Paper. Paper has the smallest increase in vehicle capacity for the outbound capacity (an increase of about $17 \%-4000 \mathrm{~kg}$ vs. $3400 \mathrm{~kg}$ - against an increase of $166 \%$ for Glass and 233\% for Plastic/Metal) what favours the assignment of more sites to the sorting station to avoid the outbound transportation (as it will imply a greater distance to be travelled as the vehicle capacity is small). For those sites assigned to the sorting station (about $70 \%$ of all sites), closed routes are defined in order to avoid the outbound transportation. For the remaining sites, if open routes were defined, the increase in the outbound transportation would not be compensated by the decrease of the inbound transportation by defining open routes.

For the remaining two materials, the open routes are to be redefined into closed ones by formulation 1.2 in Fig. 5. As final result, Glass has 17 routes, Paper 21 and Plastic/Metal 12 routes, all closed ones.

The service areas produced for the three materials are shown in Fig. 8.

As different service areas are defined, and Scenario 1 requires equal service areas among the three materials, module 2 is run for the unclear sites. When the three service areas are overlapped, the unclear sites are highlighted (see Fig. 9(a)). 101 unclear sites are identified: 8 sites are unclear between depot 231 and depot 233 , 20 sites between depots 232 and 235, 28 sites between depots 234 and 235 and the remaining 45 sites are unclear between depots 233 and 235. The Multi-Product, MDVRP formulation was able to solve the sub-problems with 8,20 and 28 unclear sites. However, the subproblem with 45 unclear sites has to be solved by the heuristic rule. The final service areas for Scenario 1 are built as shown in Fig. 9(b). 


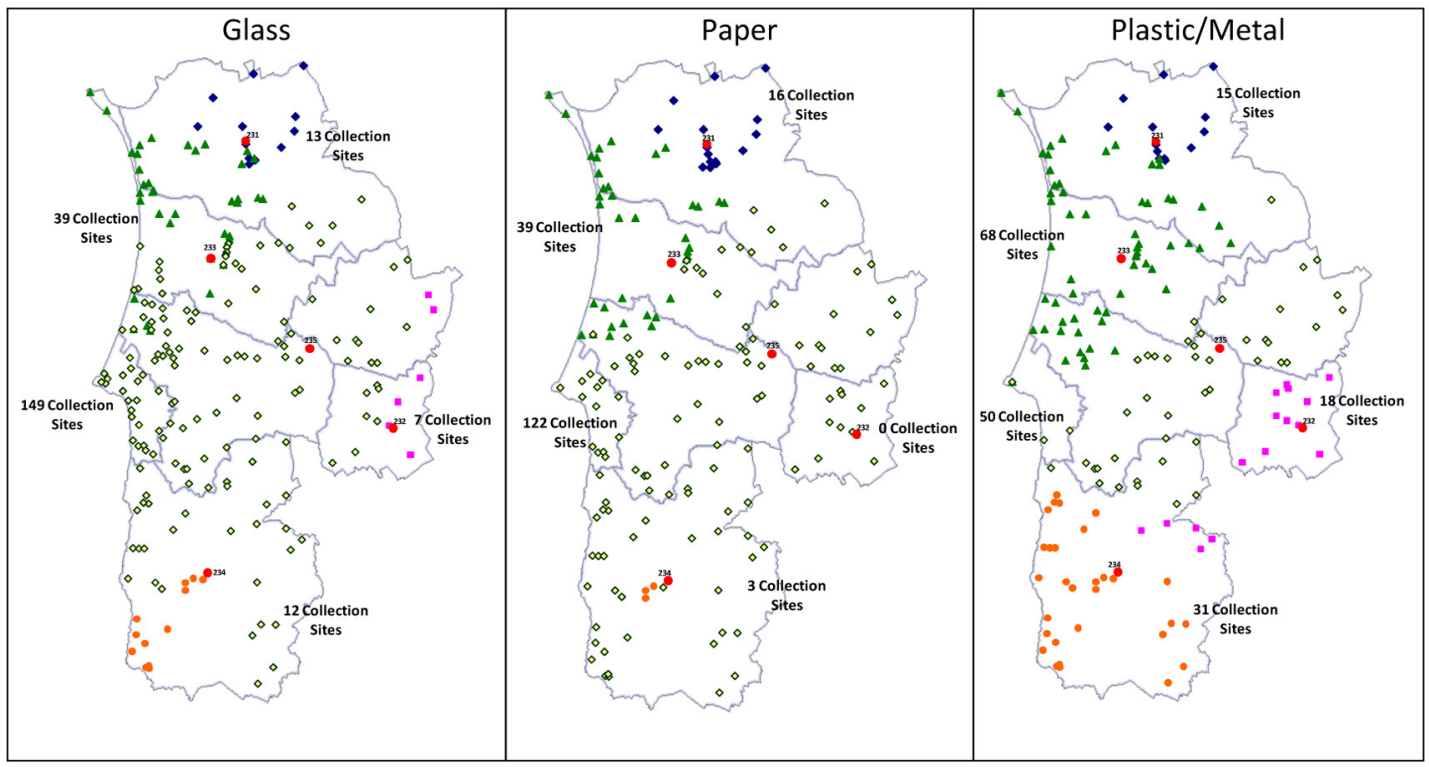

Fig. 8. Service areas obtained for each packaging material.

Ramos et al. (2014).

Having the service areas defined, module 3 is applied to establish the final collection routes for each depot and each packaging material. As a result, one obtains 32 routes to collect Glass, 21 to collect Paper and 49 to collect Plastic/Metal. The total distance travelled in the planning horizon is $24,405 \mathrm{~km}$ (see Ramos et al. (2014) for the computational results).

Module 4 is then applied to schedule the 102 collection routes. It is assumed a minimal time interval $\left(I_{m}\right)$ to collect Paper of 4 days, for Plastic/Metal 9 days and for Glass 20 days, and a maximum interval $\left(A_{m}\right)$ of 5,10 and 20 days, respectively. Given the service areas obtained and considering that vehicles are fixed at depots,
9 vehicles are required, with the following distribution by depot (Fig. 10).

The nine vehicle schedules are shown in Fig. 11, where the usage time (in minutes) is identified for each day of the planning horizon. It can be seen that vehicle 4 , based at depot 234 , has the lowest usage rate since this depot is responsible to collect only three collection sites (see Fig. 9(b)). Two vehicles are assigned to depot 233: vehicle 2 operates 3950 min per month, with a usage rate of $41 \%$ ( $3950 \mathrm{~min} /(20$ days $\times 480 \mathrm{~min}))$; while vehicle 3 operates $5632 \mathrm{~min}$, with a usage rate of $59 \%$. Given the sum of the usage rates, a macro analysis could conclude that the workload of depot

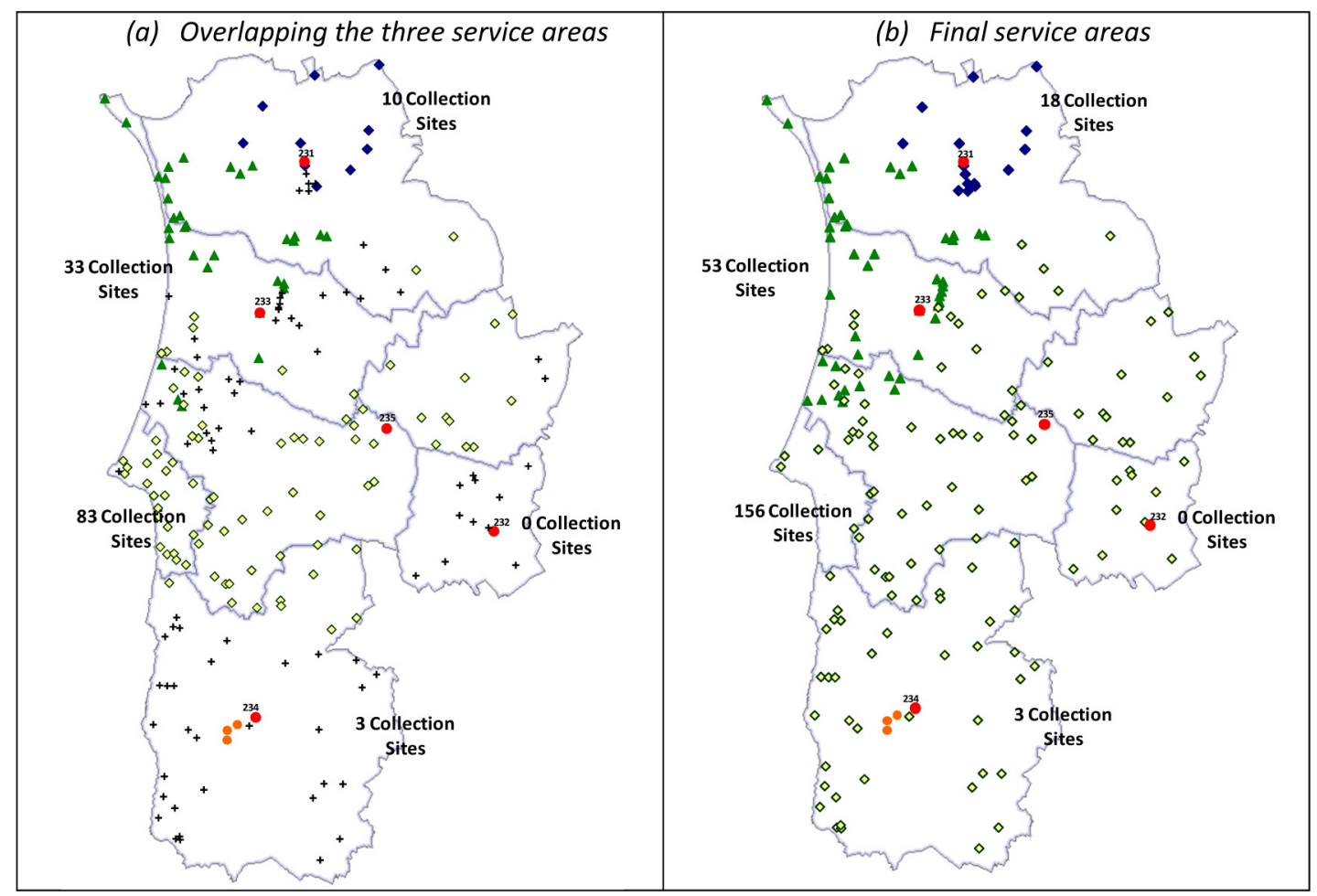

Fig. 9. (a) Service areas overlapped and (b) final service areas. 


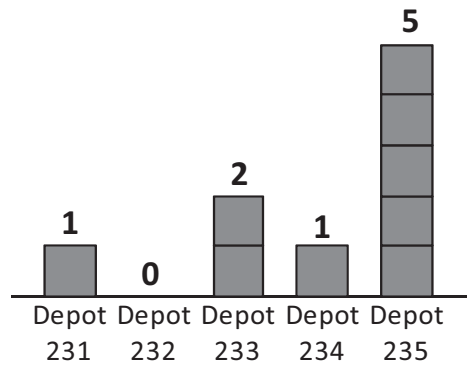

Fig. 10. Vehicle fleet distribution among depots in scenario 1.

Table 2

Computational results for module $4 \mathrm{a}$ in scenario 1 .

\begin{tabular}{lllll}
\hline & $\begin{array}{l}\text { Number of } \\
\text { variables }\end{array}$ & $\begin{array}{l}\text { Number of } \\
\text { constraints }\end{array}$ & $\begin{array}{l}\text { Running } \\
\text { time (s) }\end{array}$ & Gap (\%) \\
\hline Module 4a & 36,541 & $1,382,831$ & 126 & 0 \\
\hline
\end{tabular}

233 requires only one vehicle. However, given the routes duration and the maximum duration of a working day, it is not possible to perform all routes with a single vehicle. For depot 235, which is responsible to collect 156 sites, five vehicles are needed. These vehicles are used every day of the planning horizon and the minimum usage rate among them is $74 \%$ (vehicle 8 ) and the maximum is $86 \%$ (vehicle 9). Note that Depot 235 act also as the sorting station and, therefore, a larger number of collection sites were assigned to it.

The results presented by module $4 \mathrm{a}$ are optimal ones given the set of routes defined by the previous module. The scheduling model run in $126 \mathrm{~s}$ and has an optimal value of $24,405 \mathrm{~km}$. See Table 2 for the computational results.

\subsubsection{Scenario 2: service areas by recyclable material}

In this scenario, service areas are defined by packaging material and are obtained after module 1, as it is shown in Fig. 8. The service

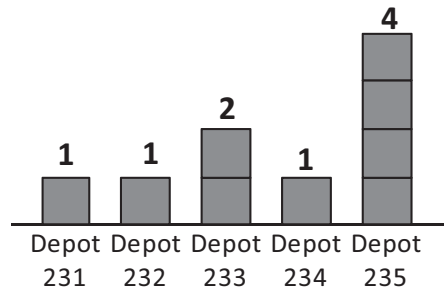

Fig. 12. Vehicle fleet distribution among depots in scenario 2.

areas are quite different between recyclable materials. For instance, depot 234 is responsible to collect 12 sites for material Glass, but only 3 sites for Paper and 31 sites for Plastic/Metal; depot 232 does not have any site assigned where Paper should be collected, while for Glass and Plastic/Metal, a total of 7 and 18 sites are assigned, respectively.

Module 3 is applied to each depot and each packaging material to define the final collection routes. A solution with a total of $23,294 \mathrm{~km}$ is obtained, where 21 routes are created to collect Paper, 50 to collect Plastic/Metal and 32 to collect Glass. The computational results can be seen at Ramos et al. (2014).

Route scheduling is done by module $4 \mathrm{a}$, where the routes are assigned to a day in the planning horizon and to a vehicle, assuming that sharing resources are not allowed. In this scenario, 9 vehicles are also required, but with a different distribution by depot (see Fig. 12).

Scheduling results for each vehicle are shown in Fig. 13. Vehicle 1 , based at depot 231 , has a usage rate of $42 \%$; vehicle 2 , based at depot 232 , has the lowest usage rate of $18 \%$ since it is again the depot with lowest number of collection sites assigned; depot 233 needs two vehicles (vehicle 3 and 4 ) with a usage rate of $61 \%$ and $44 \%$, respectively; vehicle 5 , based at depot 234 , has a usage rate of $34 \%$; finally, depot 235 has four vehicles, all of them with a high usage rate $(83 \%, 78 \%, 87 \%$ and $85 \%)$.

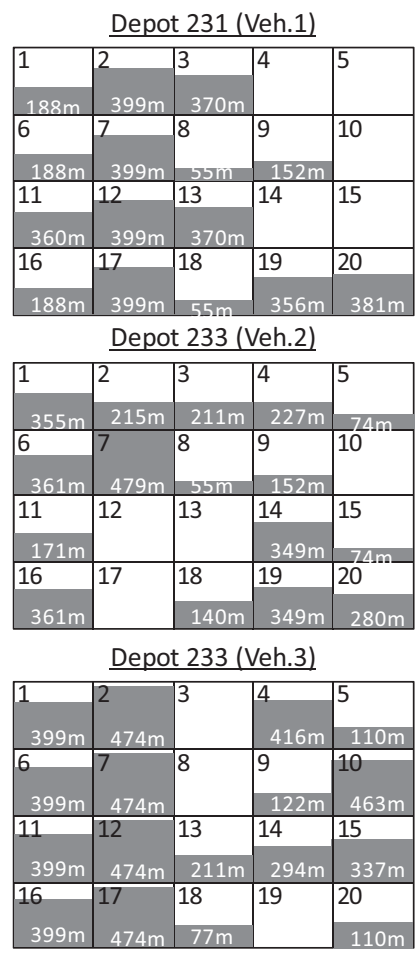

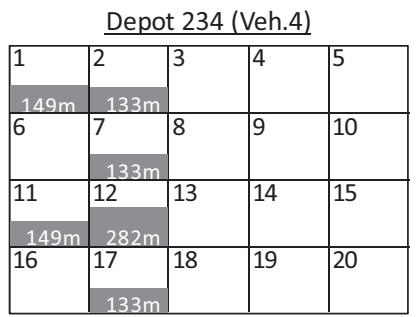

Depot 235 (Veh.5)



Depot 235 (Veh.6)

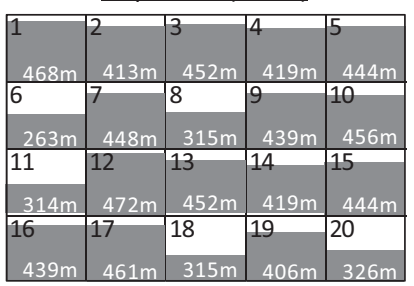

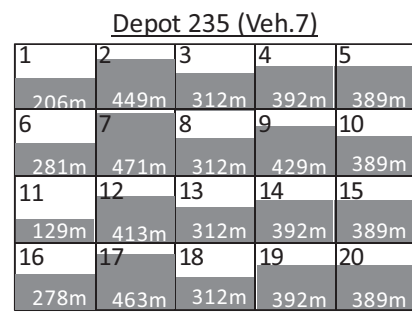

Depot 235 (Veh.8)

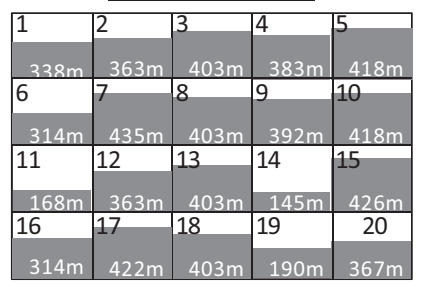

Depot 235 (Veh.9)

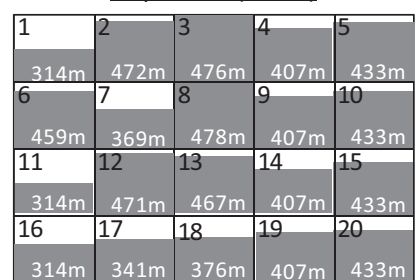

Fig. 11. Schedule by vehicle in scenario 1 . 


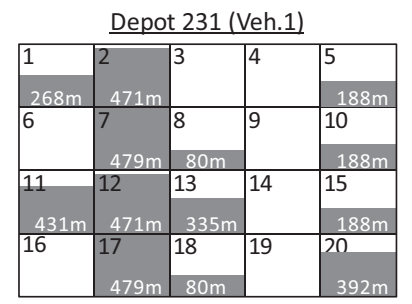

Depot 232 (Veh.2)

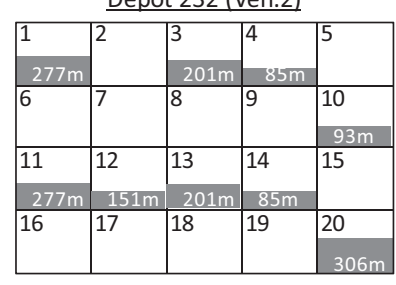

Depot 233 (Veh.3)

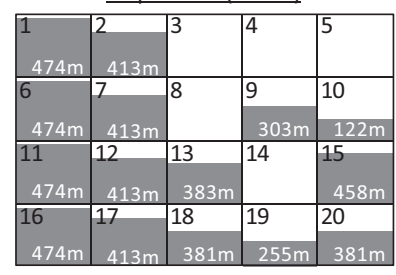

Depot 233 (Veh.4)



Depot 234 (Veh.5)

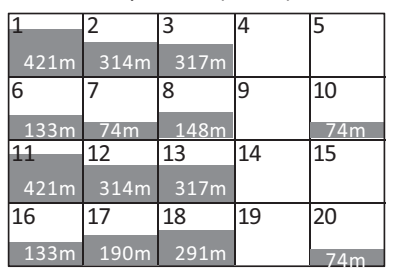

Depot 235 (Veh.6)

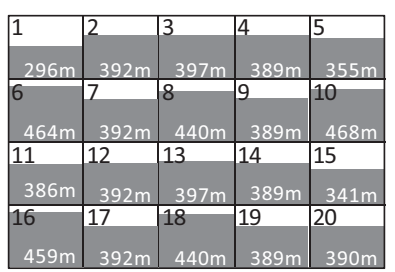

Depot 235 (Veh.7)



Depot 235 (Veh.8)

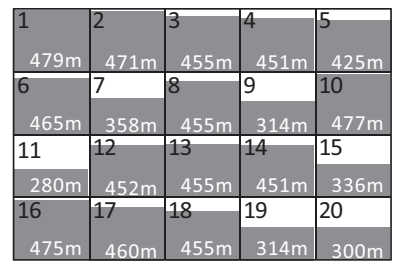

Depot 235 (Veh.9)

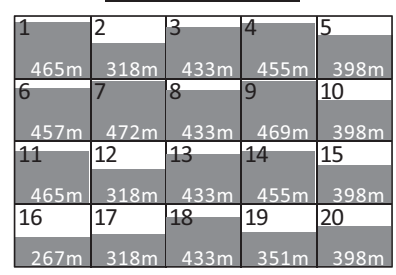

Fig. 13. Schedule by vehicle in scenario 2 .

Table 3

Computational results for module 4 a in scenario 2 .

\begin{tabular}{lllll}
\hline & $\begin{array}{l}\text { Number of } \\
\text { variables }\end{array}$ & $\begin{array}{l}\text { Number of } \\
\text { constraints }\end{array}$ & $\begin{array}{l}\text { Running } \\
\text { time (s) }\end{array}$ & Gap (\%) \\
\hline Module 4a & 25,921 & 838,483 & 98 & 0 \\
\hline
\end{tabular}

The results presented by module $4 \mathrm{a}$ are the optimal ones given the set of routes defined by the previous module. The scheduling model run in $98 \mathrm{~s}$ and has an optimal value of $23,294 \mathrm{~km}$ (see Table 3).

\subsubsection{Scenario 3: sharing resources}

In this scenario, service areas and vehicle routes are the ones defined for scenario 2 . The main difference between scenarios 2 and 3 concerns the scheduling module: vehicles based in one depot can perform closed routes of other depots in scenario 3. In this case, it is expected that the distance travelled will increase because the vehicles have to move between depots, but the number of vehicles will decrease.

We test a solution with eight vehicles, distributed by depot as shown in Fig. 14(a), and the total distance travelled increases to $23,421 \mathrm{~km}$ (more $0.6 \%$ comparing with the previous scenario). A solution with seven vehicles was also tested (see Fig. 14(b)), and the (a) 8 vehicles

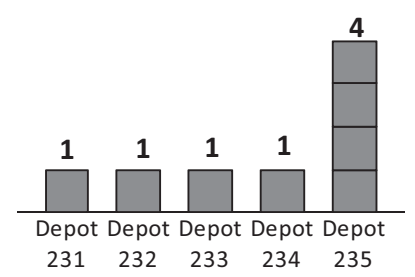

(b) 7 vehicles

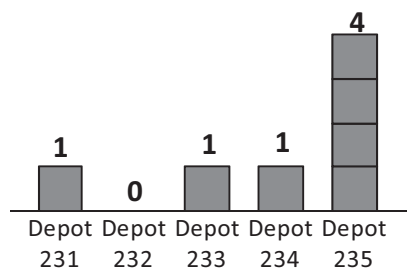

Fig. 14. Vehicle fleet distribution among depots in scenario 3 with (a) 8 and (b) 7 vehicles. total distance obtained is now $24,198 \mathrm{~km}$ (more $3.9 \%$ than scenario 2 ). No integer solution is obtained if only six vehicles are available.

The vehicle schedules for the seven-vehicle solution are shown at Fig. 15. In this solution five out of the seven vehicles have high usage rates. Vehicle 2, based at depot 233, has the highest rate, $95 \%$. Comparing with the previous scenario, depot 233 has one less vehicle, meaning that a higher usage rate is achieved. The remaining routes from depot 233 are now performed by vehicles based at depot 231 and 235, contributing for a higher usage rate of those vehicles. Vehicles based at depot 235 have usage rates varying from $87 \%$ to $91 \%$. Besides performing routes from depot 235 , these vehicles execute routes assigned to depot 233 , as mentioned, and to depot 232, since in this solution no vehicles have been based in that depot. Vehicle 1, based at depot 231 , has a usage rate of $51 \%$, nine percent higher than in the previous scenario, since it also performs routes of depot 233 . This is also the case with vehicle 3 , based at depot 234 , which now increases its usage rate to $37 \%$ (34\% in the previous scenario) as it performs routes assign to depot 232 .

Fig. 16 illustrates the routes performed by vehicle 1 , based at depot 231, on days 4 and 14 . In those days, vehicle 1 has to travel to depot 233, perform route 104 to collect Plastic/Metal, unload at depot 233, and then return to its home depot. In those days, the vehicle 1 is used during $429 \mathrm{~min}$.

In terms of computational results, module $4 \mathrm{~b}$ has not been able to prove optimality within the time limit of $1 \mathrm{~h}$, but a low gap is obtained (see Table 4).

\subsubsection{Scenario 4: open routes between depots}

Scenario 4 breaks up with all four practices mentioned, meaning that open routes are allowed between depots. In scenario 3 , vehicles

Table 4

Computational results for module $4 \mathrm{~b}$ in scenario 3 with seven vehicles.

\begin{tabular}{lllll}
\hline & $\begin{array}{l}\text { Number of } \\
\text { variables }\end{array}$ & $\begin{array}{l}\text { Number of } \\
\text { constraints }\end{array}$ & $\begin{array}{l}\text { Running } \\
\text { time (s) }\end{array}$ & Gap (\%) \\
\hline Module 4b & 33,603 & $1,129,857$ & 3600 & 0.3 \\
\hline
\end{tabular}



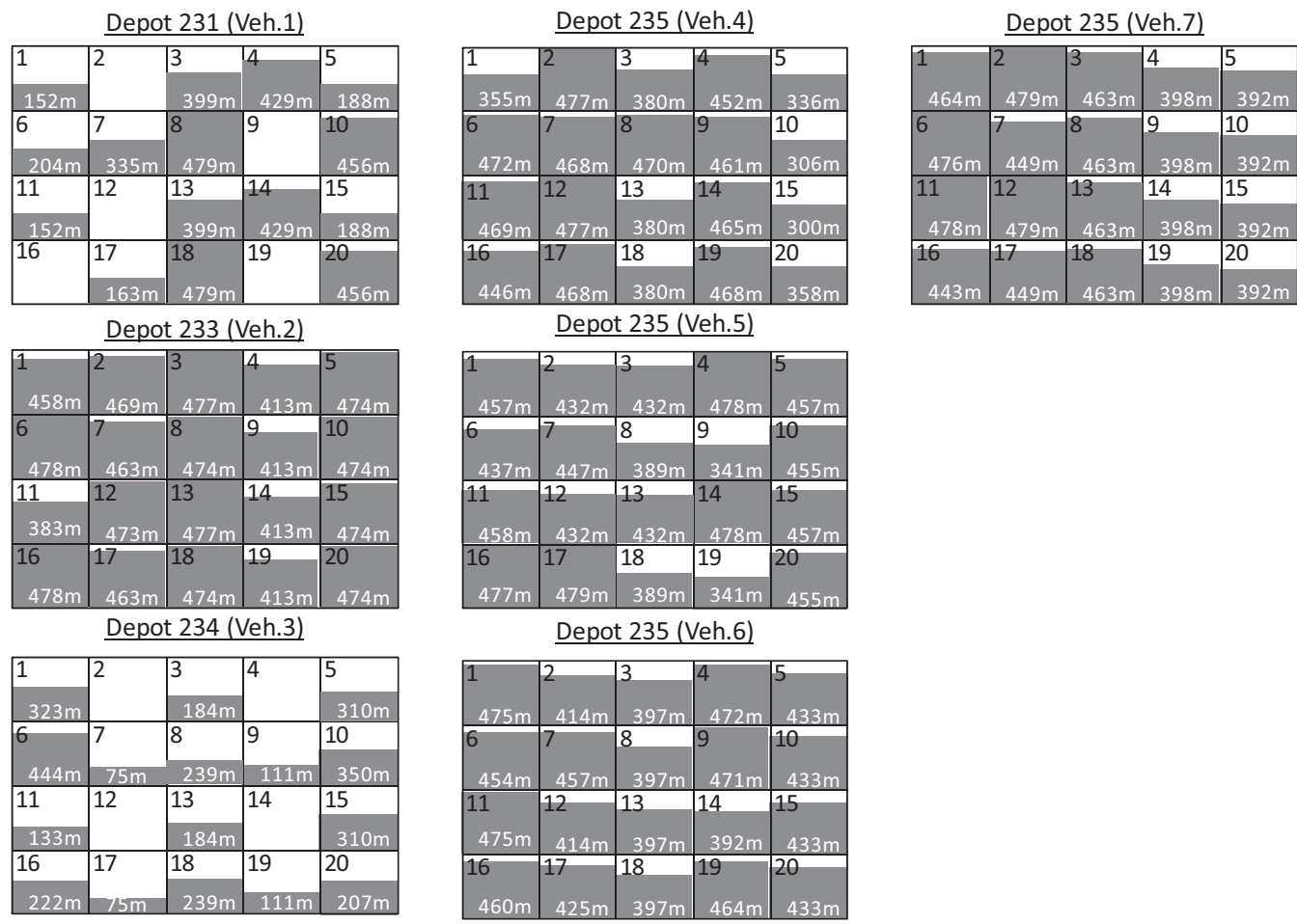

Fig. 15. Schedule by vehicle in scenario 3.

can be shared among depots but they travel empty between depots. In scenario 4 , the main idea is to minimize the number of those empty routes. In order to do so, advantage will be taken from the open routes between depots generated at module 1 which allow the vehicle relocation. Therefore, the relocating movements can now be collection routes and not only empty routes. The scheduling module 4c considers routes generated by modules 1 and 3, which are closed and open routes, and selects the ones that should take part in the final schedules.

The total distance decreases when compared with the previous scenario. With eight vehicles, a total distance of $23,181 \mathrm{~km}$ is achieved, while a total of $23,687 \mathrm{~km}$ is obtained if seven vehicles are used.

Each of the seven vehicles schedules is shown at Fig. 17. Vehicle 1 is now operating $4077 \mathrm{~min}$, corresponding to $42 \%$ of usage rate. Vehicle 2 has a usage rate of 92\%, while vehicle 3 has increased its usage rate to $47 \%$ ( $37 \%$ in the previous scenario) since it performs open routes between depots 234, 232 and 235. The four vehicles based at depot 235 maintain high usage rates (from $85 \%$ to $91 \%$ ).

Fig. 18 shows the routes performed by vehicle 3 , based at depot 234 , on days 1 and 11 to illustrate the sharing resources allowed

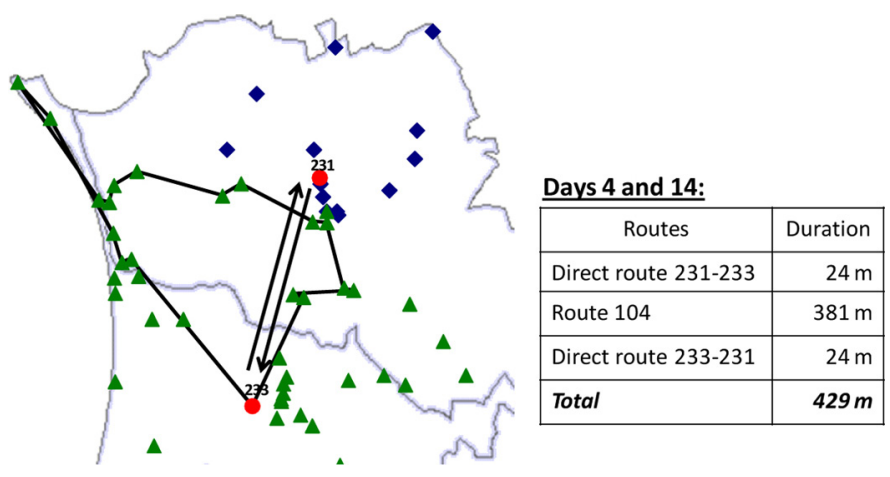

Fig. 16. Illustration of the routes performed by vehicle 1 on days 4 and 14 . by considering open routes. One closed route and two open routes between depot 234 and depot 232 are performed by this vehicle on these days.

Concerning the computational results for module $4 \mathrm{c}$ with seven vehicles (see Table 5), one should say that optimality has not been proven within the time limit of $1 \mathrm{~h}$, but a low gap is obtained (0.3\%).

\subsection{Cost analysis}

As mentioned in the previous section, the obtained solutions vary between $23,181 \mathrm{~km}$ and 24,405 $\mathrm{km}$ and between 7 and 9 vehicles. Table 6 summarizes the results for the scenarios studied. It shows that scenario $4 \mathrm{a}$ is the scenario with the lowest distance travelled per month $(23,181 \mathrm{~km})$ while scenarios $3 \mathrm{~b}$ and $4 \mathrm{~b}$ require the lowest number of vehicles and drivers (7).

To compare those solutions a cost-analysis is performed. Three main costs will be computed for each solution: fuel costs, vehicles depreciation costs and driver's costs. The fuel costs are a linear function of the distance travelled. It has been estimated $0.5 €$ per

Table 5

Computational results for module $4 \mathrm{c}$ in scenario 4 with seven vehicles.

\begin{tabular}{lllll}
\hline & $\begin{array}{l}\text { Number of } \\
\text { variables }\end{array}$ & $\begin{array}{l}\text { Number of } \\
\text { constraints }\end{array}$ & $\begin{array}{l}\text { Running } \\
\text { time (s) }\end{array}$ & Gap (\%) \\
\hline Module 4c & 38,041 & $1,241,843$ & 3600 & 0.3 \\
\hline
\end{tabular}

Table 6

Results for each scenario.

\begin{tabular}{llll}
\hline Scenario & Distance & No. vehicles & No. drivers \\
\hline 1 & 24,405 & 9 & 9 \\
2 & 23,294 & 9 & 9 \\
3a & 23,421 & 8 & 8 \\
3b & 24,198 & 7 & 7 \\
$4 \mathrm{a}$ & 23,181 & 8 & 8 \\
4b & 23,687 & 7 & 7 \\
\hline
\end{tabular}




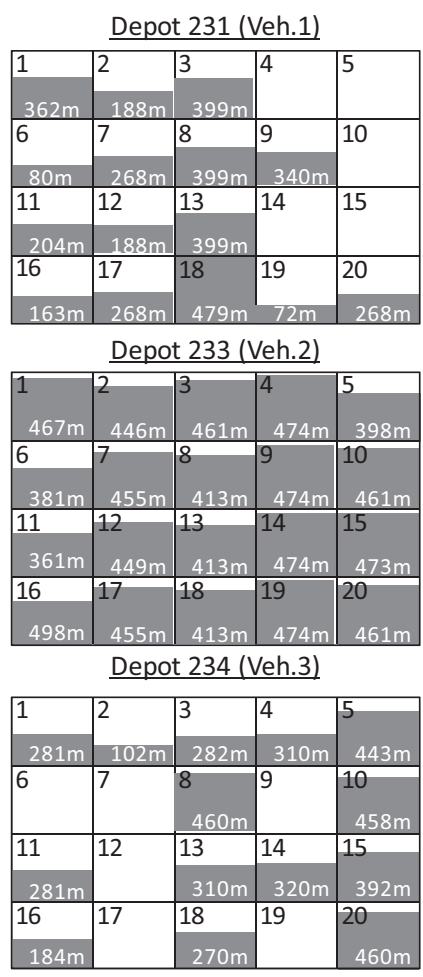

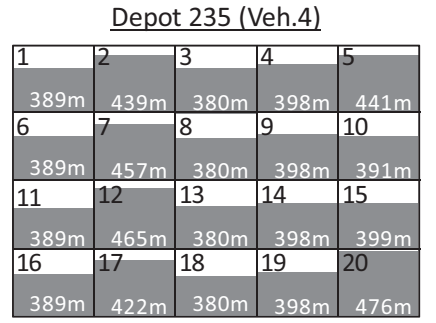
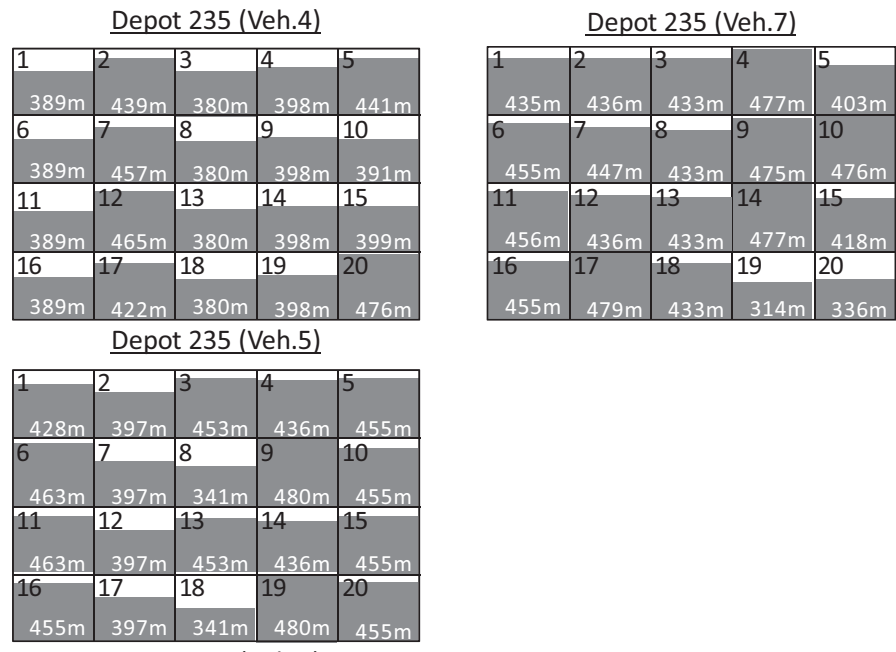

Depot 235 (Veh.6)

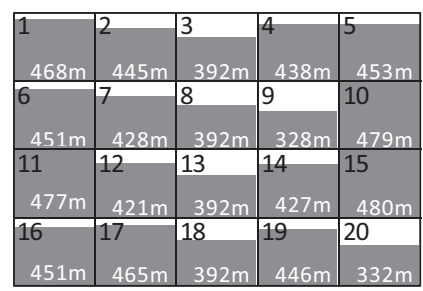

Fig. 17. Schedule by vehicle in scenario 4 kilometer driven. Vehicle depreciation is estimated considering vehicles acquisition cost and the useful life assumed for taxes purpose. In particular, the acquisition cost is about $100,000 €$ and the useful life is of 5 years, which leads to an annual depreciation of $20,000 €$. The drivers costs are estimated in $900 €$ per month, paid 14 months per year, and it is considered one driver per vehicle.

Fig. 19 depicts the total cost for the current solution and for each scenario studied. Scenario 1 has $12.8 \%$ less distance travelled than in the current solution and the total annual cost decreases 5\% if the municipalities' boundaries are not respected when defining the service areas. Comparing scenario 2 (service areas are defined by packaging material) to scenario 1 (service areas by depot), the distance travelled decreases $5 \%$, while the required number of vehicles is maintained. The total cost decreases in $1.7 \%$ when compared to scenario 1 and $6.4 \%$ when compared to the current solution. The largest decrease in the total cost is obtained in the scenario where resources are shared among depots (scenarios 3 and 4). In scenario $3 \mathrm{~b}$, the total cost decreases $13.3 \%$ regarding scenario 2 and

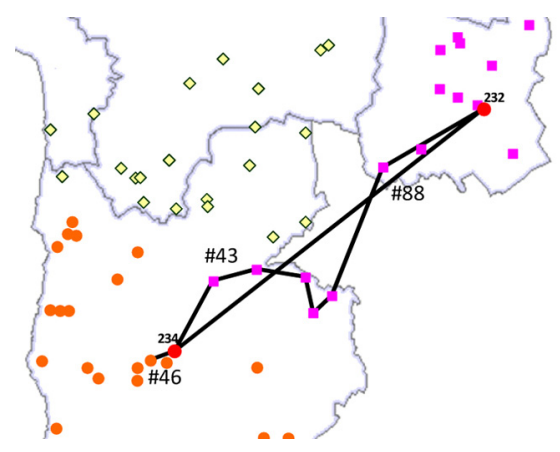

Days 1 and 11:
\begin{tabular}{|l|r|}
\hline \multicolumn{1}{|c|}{ Routes } & Duration \\
\hline Route 46 & $74 \mathrm{~m}$ \\
\hline Route 43 & $150 \mathrm{~m}$ \\
\hline Route 88 & $57 \mathrm{~m}$ \\
\hline Total & $\mathbf{2 8 1} \mathbf{~ m}$ \\
\hline
\end{tabular}

Fig. 18. Illustration of the routes performed by vehicle 3 on days 1 and 11 .
18.9\% when compared to the current solution. These gains come from sharing vehicles among depots which allows the reduction of the number of vehicles to seven. When open routes between depots are allowed, the total distance travelled decreases even more $(23,687 \mathrm{~km}$ against $24,198 \mathrm{~km}$, about $2 \%)$ and the total cost decreases about $20 \%$ when comparing with the current solution.

Significant savings are obtained when some of the current practices are removed. However, from an operations management perspective, it is more complex to manage scenario 4, where three different service areas, shared vehicles and open routes have to be dealt with, than the current solution where each depot has one service area, with vehicles assigned and only closed routes, which allows each depot to act independently. However, these latter practices lead to a larger travelled distance and more resources, as vehicles and drivers. In the breakthrough scenario (scenario 4 ), all depots and vehicles are integrated and act as part of the same system. This kind of solution decreases the distance travelled and the resources needed but demands a decision support

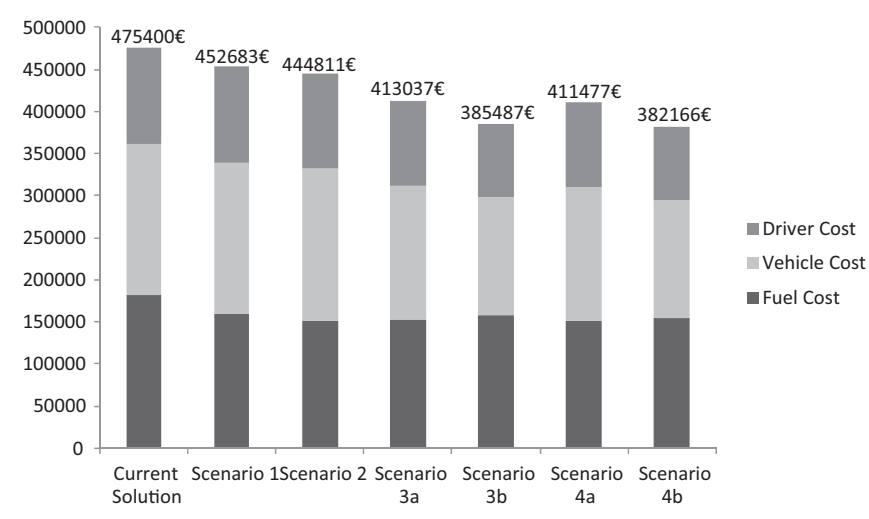

Fig. 19. Total cost for each scenario studied. 
system to help managing the increased complexity. The solution methodology developed in this work can be seen as a main pillar towards the development of a decision support system, supporting the routes definition and vehicle schedules under an innovative scenario.

\section{Conclusions}

In this paper four practices currently used by recyclable collection systems operating in Portugal have been analysed so as to propose improvements on their setups. Given that the systems in study have multiple depots managed independently, the main idea was to explore management operations in an integrated way so that gains in terms of efficiency could be achieved, by decreasing the total operational costs.

To achieve this goal this paper developed a unified solution methodology that is capable to plan the collection systems exploring different practices. This solution methodology is applied to a real packaging waste collection system. Four scenarios were analysed, developed by cumulatively removing each of the four practices. The problems solved are the Multi-Product, Multi-Depot Periodic Vehicle Routing Problem, the Multi-Depot Periodic Vehicle Routing Problem, the Multi-Depot Periodic Vehicle Routing Problem with Shared Resources and Multi-Depot Periodic Vehicle Routing Problem with Inter-Depot Routes. The solution methodology defines service areas and vehicle routes in an integrated way. Afterwards it assigns the routes to a day on the planning horizon according to each scenario.

Each scenario was evaluated in terms of travelled distance and number of required vehicles. Also the total annual cost of each scenario considering fuel costs, vehicle depreciation costs and driver's costs, was calculated. It was concluded that by removing the practice of not sharing resources among depots implies the highest impact on the total cost, since sharing resources enables a decrease up to two vehicles. Moreover, defining service areas by recyclable material instead of depot (scenario 2 vs. scenario 1 ) leads to a positive impact of $1.7 \%$ on the total cost. Sharing resources instead of having the vehicle fixed at depots (scenario 3 vs. scenario 2) conducted to a positive impact of $13.3 \%$ in total cost (the highest impact, as mentioned). Performing mixed closed and open routes instead of allowing only for closed routes (scenario $4 \mathrm{vs}$. scenario 3 ) resulted in a positive impact of $0.9 \%$ in total cost. Finally, comparing the current solution with the innovative scenario (scenario 4 , where all of the four practices are removed), a decrease of $20 \%$ in the total cost was observed.

It is a fact that each scenario studied increases the complexity of managing operations within the recyclable collection systems, but significant costs savings have been attained. Moreover, the current situation is driven by management options adopted by political issues which led to the use of municipal boundaries when planning the systems. Such practice proved to diminish the system's efficiency and consequently alternative solutions should be pursued. The improved results need, however, to be demonstrated and discussed with the management board. This was achieved, in this work, through a close collaboration between the research and the company management teams.

As future work and still aiming to further improving operation, other alternative operational practices can be studied. Such as the night collection in opposition to the daytime collection, the latter in use in the packaging collection system under study. Notice that performing a daytime collection activity may lead to a decrease in the overall system efficiency given traffic congestion (that may slow down collection). Night collection can, however, increase the collection efficiency in tradeoff with an increase of personnel costs.
The solution methodology proposed defines firstly the service areas and vehicle routes in an integrated way, while the scheduling decisions are taken in a second phase. This sequential method causes a low usage rate of some vehicles, representing a pitfall of the proposed methodology. A tighter integration of those decisions would lead to higher usage rates, and therefore, better results. As future research, the solution methodology should be improved to tackle the three decisions simultaneously. In addition, it would also be interesting to study this problem by applying different techniques, such as meta-heuristics, making a comparison of the results obtained. Finally, another future work direction is to apply this methodology to other packaging collection systems in order to quantify the results of more case studies so as to corroborate the conclusions of the current work.

\section{Acknowledgements}

The insightful contributions of the anonymous referees are gratefully acknowledged.

This work was partially supported by the CMA/FCT/UNL under the project PEst OE/MAT/UI0297/2011.

\section{References}

Alonso F, Alvarez MJ, Beasley JE. A tabu search algorithm for the periodic vehicle routing problem with multiple vehicle trips and accessibility restrictions. J Oper Res Soc 2008;59:963-76

Anghinolfi D, Paolucci M, Robba M, Taramasso AC. A dynamic optimization model for solid waste recycling. Waste Manage 2013;33:287-96.

APA. Avaliação dos custos dos SMAUTs com as operações de recolha e triagem; 2008 http://www.tratolixo.pt/Comunicacao/Paginas/equilibrioValoresContra.aspx [accessed 26.10.12].

Baldacci R, Hadjiconstantinou E, Mingozzi A. An exact algorithm for the capacitated vehicle routing problem based on a two-commodity network flow formulation. Oper Res 2004;52:723-38.

Baldacci R, Mingozzi A. A unified exact method for solving different classes of vehicle routing problems. Math Program 2009;120:347-80.

Beltrami EJ, Bodin LD. Networks and vehicle routing for municipal waste collection. Networks 1974;4:65-94.

Chao IM, Golden BL, Wasil E. An improved heuristic for the period vehicle-routing problem. Networks 1995;26:25-44.

Christofides N, Beasley JE. The period routing problem. Networks 1984;14:237-56

Cordeau JF, Gendreau M, Laporte G. A tabu search heuristic for periodic and multidepot vehicle routing problems. Networks 1997;30:105-19.

Craighill AL, Powell JC. Lifecycle assessment and economic evaluation of recycling: a case study. Resour Conserv Recy 1996;17:75-96.

Crevier B, Cordeau JF, Laporte G. The multi-depot vehicle routing problem with interdepot routes. Eur J Oper Res 2007;176:756-73.

Faccio M, Persona A, Zanin G. Waste collection multi objective model with real time traceability data. Waste Manage 2011;31:2391-405.

Gaudioso M, Paletta G. A heuristic for the periodic vehicle-routing problem. Transp Sci 1992;26:86-92.

Golden BL, Magnanti TL, Nguyen HQ. Implementing vehicle routing algorithms Networks 1977:7:113-48.

Golden B, Raghavan S, Wasil E, editors. Vehicle routing problem: latest advances and new challenges. New York: Springer; 2008.

Hadjiconstantinou E, Baldacci R. A multi-depot period vehicle routing problem arising in the utilities sector. J Oper Res Soc 1998;49:1239-48.

Johansson OM. The effect of dynamic scheduling and routing in a solid waste management system. Waste Manage 2006;26:875-85

Laporte G. Fifty years of vehicle routing. Transp Sci 2009;43:408-16.

Laporte G, Nobert Y, Arpin D. Optimal solutions to capacitated multi-depot vehicle routing problems. Congr Numer 1984;44:283-92.

Laporte G, Nobert Y, Taillefer S. Solving a family of multi-depot vehicle-routing and location-routing problems. Transp Sci 1988;22:161-72.

Lau HCW, Chan TM, Tsui WT, Pang WK. Application of genetic algorithms to solve the multidepot vehicle routing problem. IEEE Trans Autom Sci Eng 2010;7:383-92.

Lim A, Wang F. Multi-depot vehicle routing problem: a one-stage approach. IEEE Trans Autom Sci Eng 2005;2:397-402

Marques RC, da Cruz NF, Carvalho P. Assessing and exploring (in)efficiency in Portuguese recycling systems using non-parametric methods. Resour Conserv Recy 2012;67:34-43.

Mourgaya M, Vanderbeck F. Column generation based heuristic for tactical planning in multi-period vehicle routing. Eur J Oper Res 2007;183:1028-41

Parthanadee P, Logendran R. Periodic product distribution from multi-depots under limited supplies. IIE Trans 2006;38:1009-26.

Ramos TRP, Gomes MI, Barbosa-Povoa AP. Planning waste cooking oil collection systems. Waste Manage 2013;33:1691-703 
Ramos TRP, Gomes MI, Barbosa-Povoa AP. Economic and environmental concerns in planning recyclable waste collection systems. Transp Res E 2014;62:34-54.

Russel R, Igo W. An assignment routing problem. Networks 1979;9:1-17.

Salhi S, Sari M. A multi-level composite heuristic for the multi-depot vehicle fleet mix problem. Eur J Oper Res 1997;103:95-112.

SPV. Caracterização dos Sistemas Municipais Aderentes ao Sistema Ponto Verde; 2011 http://www.spvnet.net/2011/data/smauts/carac_smaut.pdf [accessed 28.08.12].
Teixeira J, Antunes AP, de Sousa JP. Recyclable waste collection planning - a case study. Eur J Oper Res 2004;158:543-54.

Thangiah SR, Salhi S. Genetic clustering: an adaptive heuristic for the multidepot vehicle routing problem. Appl Artif Intell 2001;15:361-83.

Vidal T, Crainic TG, Gendreau M, Lahrichi N, Rei W. A hybrid genetic algorithm for multidepot and periodic vehicle routing problems. Oper Res 2012;60: 611-24. 